\title{
Analysis of Alzheimer Symptoms and Stages Using Canny Edge Detector in Image Segmentation
}

\author{
M.Jainab., M. Phil ${ }^{1}$ Prof. B. Mohamed Faize Basha, MCA., M.Phil., ${ }^{2}$ Prof. M. \\ Mohamed Surpudeen, M.sc., M. Phil., \\ 1 (Department of Computer Science, Jamal Mohamed College, India) \\ 2(Department of Computer Science,JamalMohamedCollege,India)
}

\begin{abstract}
Alzheimer's disease is the most common form of dementia.It is a neurological brain disorders. The hippocampus is known to shrink in time due to cell death,and it is linked with increased memory loss, which is a primary symptom of AD.In previous work, the active shape model is use to represent the shape of hippocampus, this model does not accomplish the exact volume of hippocampus. In this paper segmentation done on the hippocampusregion by the canny edge detector algorithm.This algorithm is used to trace the edges of hippocampus region in MRI images and hysteresis thresholding is used to trace the image and the segmented image is measured using voxel based morphometric technique for better result.
\end{abstract}

Keywords: AD,MRI, Hippocampus, voxel

\section{Introduction}

Alzheimer's disease is a neurological brain disorder named after a German physician. Alzheimer's disease was discovered in 1906 by Alois Alzheimer a German neurologist and psychiatrics. Alzheimer is known as the loss of mental function such as thinking, memory, and reasoning that is severe enough to interfere with a person's daily functioning. The appearance of Alzheimer Disease symptoms (AD) are resulted based on part of the brain infection or damage.

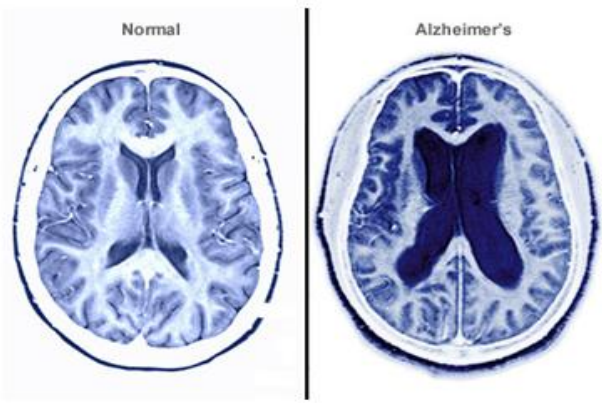

Fig1: Normal and affected brain

Hippocampus is a component with complex structure and plays a vital role in the functioning of the brain. It's primary function is related with encoding of episodic memory. It is also believed to play an important role in both encoding and retrieval of other types of long term memory. Volumetric approaches for the analysis of Hippocampal Structures (HS) are required for the diagnosis of differentdiseases. In order to diagnose, evaluate and compare patient datasets, standardized and reproducible analysis are needed in clinical routine and research

${ }^{[1]}$ MR-volumetric assessmentof temporomesial structures, especially hippocampal structures, play an important role in the diagnosis of temporal lobe epilepsy degenerative diseases like Alzheimer dementia andthe evaluation of their course of disease. Objective, reliable and reproducible semi- or fully automaticmethods are required to record minor atrophies, evaluate chronological changes and confidently compare them among different observers and institutes. Resulting increased interandintra-observer variability of manual techniques limit the role of segmentation in clinical studies. Only valid results of hippocampal volume segmentation can be confidently correlated to severity of disease progressionand to neurohistopathological results.Conventionally used manual segmentation is a time consuming procedure, which therefore cannot be performed in clinical routine. Fully automatic segmentation may sometimes fails ifthe hippocampus is relatively small and the shape of the object is highly variable. Compared to this, semiautomaticmethods, may sometimes provide a more realistic approachbecause of the combination of human expertise andautomatic techniques. The location of the hippocampus is shown in the figure 2 : 
O. T. Carmichael, H. A. Aizensteinet al ${ }^{[2]}(2005)$ did research on atlas-based registration and segmentation of the hippocampus and found out that fully deformable methods can be competitive with the human raters in terms of overlap ratio.

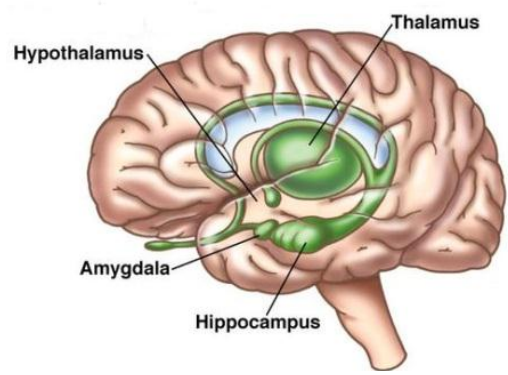

Figure 2 :View of hippocampus region in brain

For segmenting the hippocampus region canny edge detection algorithm is used here.In the segmented image, the volume of shrinkage inhippocampus region is measured using Voxel- based Morphometry. In [3], The application of voxel-level three-dimensional registration to serial MRI is described. This fluid registration brain model determines deformation fields modeling brain change, which are consistent with a model describing a viscous fluid. In this method, first, suitable values for the viscosity-body-force-ratio, $\alpha(0.01)$, and the number of iterations (300), were established and the convergence, repeatability, linearity, and accuracy are investigated by comparing the results with expert manual segmentation. The mean absolute volume difference between fluid and manual segmentation was $0.7 \%$. Fluid registration has potential importance for tracking longitudinal structural changes in brain particularly in the context of the clinical trial where large number of subjects may have multiple MR scans. In [4-11] hippocampus subfield segmentation, size estimation and features are discussed.

\section{Canny Edge Detection Algorithm:}

${ }^{[12]}$ The algorithm runs in 4 separate steps:

1. Noise Reduction: Blurring of the image to remove noise.

2. Finding gradients: The edges should be marked where the gradients of the image has large magnitude

3. Non-maximum suppression: Only local maxima should be marked as edges.

4. Tracking edges through the image and hysteresis thresholding: Final edges are determined by suppressing all edges that are not connected to a very certain (strong) edge.

Each step is described in the following subsections

\section{Step 1.Noise Reduction:} this.

Usually noise reduction implies some sort of blurring operation. Most people use a Gaussian filter to do

\section{Step 2:finding the Gradient}

Compute the derivatives $(\operatorname{Dx}(\mathrm{x}, \mathrm{y})$ and $\operatorname{Dy}(\mathrm{x}, \mathrm{y}))$ of the image in the $\mathrm{x}$ and $\mathrm{y}$ directions. You can do this exactly the same way I suggested when performing the Sobel filter.

Then compute the gradient magnitude:

$$
D=\sqrt{D_{x}^{2}(x, y)+D_{y}^{2}(x, y)}
$$

and the angle of the gradient:

$$
\theta=\arctan \left(\frac{D_{x}(x, y)}{D_{y}(x, y)}\right)
$$

Compute _' by rounding the angle _ to one of four directions $0^{\circ}, 45^{\circ}, 90^{\circ}$, or $135^{\circ}$. Obviously for edges, $180^{\circ}=0^{\circ}, 225^{\circ}=45^{\circ}$, etc. This means _ in the ranges $\left[-22.5^{\circ} \ldots 22.5^{\circ}\right]$ and $\left[157.5^{\circ} \ldots 202.5^{\circ}\right]$ would "round" to _' 
$=0^{\circ}$. For apictoral representation, each edge take on one of for colors: Here, the colors would repeat on the lower half of the circle

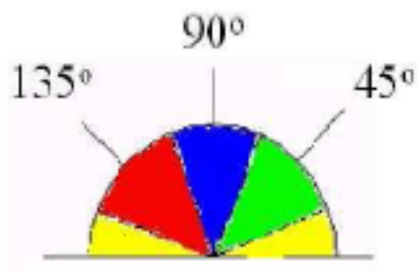

(green around $225^{\circ}$, blue around $270^{\circ}$, and red around $315^{\circ}$ ). This image taken from: http://www.pages.drexel.edu/ weg22/can tut.html

\section{Step 3: Non-Maximum Surpression}

As you might have noticed when using the Sobel filter, the edges it finds can be either very thick or very narrowdepending on the intensity across the edge and how much the image was blurred first. One would like to have edgesthat are only one pixel wide. The "non-maximal surpression" step keeps only those pixels on an edge with the highestgradient magnitude. These maximal magnitudes should occur right at the edge boundary, and the gradient magnitudeshould fall off with distance from the edge.So, three pixels in a $3 \times 3$ around pixel (x, y) are examined:

- If _' $(x, y)=0 \circ$, then the pixels $(x+1, y),(x, y)$, and $(x-1, y)$ are examined.

- If ${ }^{-}(x, y)=90^{\circ}$, then the pixels $(x, y+1),(x, y)$, and $(x, y-1)$ are examined.

- If_' $(x, y)=45^{\circ}$, then the pixels $(x+1, y+1),(x, y)$, and $(x-1, y-1)$ are examined.

- If _' $(x, y)=135^{\circ}$, then the pixels $(x+1, y-1),(x, y)$, and $(x-1, y+1)$ are examined.

If pixel $(x, y)$ has the highest gradient magnitude of the three pixels examined, it is kept as an edge. If one of the other two pixels has a higher gradient magnitude, then pixel (x,y) is not on the "center" of the edge and should notbe classified as an edge pixel

\section{Step 4.Tracing Edges through images and hysteresis thresholding :}

Large intensity gradients are more likely to correspond to edges than small intensity gradients. It is in most cases impossible to specify a threshold at which a given intensity gradient switches from corresponding to an edge into not doing so. Therefore Canny uses thresholding with hysteresis.

Thresholding with hysteresis requires two thresholds - high and low. Making the assumption that important edges should be along continuous curves in the image allows us to follow a faint section of a given line and to discard a few noisy pixels that do not constitute a line but have produced large gradients. Therefore we begin by applying a high threshold. This marks out the edges we can be fairly sure are genuine. Starting from these, using the directional information derived earlier, edges can be traced through the image. While tracing an edge, we apply the lower threshold, allowing us to trace faint sections of edges as long as we find a starting point.

Once this process is complete we have a binary image where each pixel is marked as either an edge pixel or a non-edge pixel. From complementary output from the edge tracing step, the binary edge map obtained in this way can also be treated as a set of edge curves, which after further processing can be represented as polygons in the image domain.

\section{Voxel Based Morphometry:}

[13]Voxel-based morphometry (VBM) is a neuroimaging analysis technique that allows investigation of focal differences in brain anatomy, using the statistical approach of statistical parametricmapping. In traditional morphometry, volume of the whole brain or its subparts is measured by drawing regions of interest (ROIs) on images from brain scanning and calculatingthe volume enclosed. However, this is time consuming and can only provide measures of rather large areas. Smaller differences in volume may be overlooked. VBMregisters every brain to a template, which gets rid of most of the large differences in brain anatomy among people. Then the brain images are smoothed so that each representsthe average of itself and its neighbors. Finally, the image volume is compared across brains at every voxel. 


\section{Conclusion:}

The hippocampus is segemented with canny edge detector this method need the training set from which the approximate shape of hippocampus is to be estimated.In the previous work they didn't calculate the volume of hippocampus . Our preliminary results demonstrate that the proposed approach can produce good segmentation results to find the volume of hippocampus .using this accurate result of hippocampus the medical field can easy find the volume of memory loss of Alzheimer patient .

\section{References:}

[1]. K. Somasundaram , and S. Vijayalakshmi A Novel Method for Segmentationof the HippocampusGandhigram Rural Institute, Deemed University, Gandhigram, Dindigul,Tamil Nadu(2010)

[2] O. T. Carmichael. A. Aizenstein, S. W. Davis. T. Becker. M. Thompson. C. Meltzer. Liu. Atlas-based hippocampus segmentation in Alzheimer's disease and mild cognitive impairment. NeuroImage, 27: 979-990, 2005

[3] William R. Crum, Rachael I. Scahill, Nick C. Fox, (2001), "Automated Hippocampal Segmentation by Regional Fluid Registration of Serial MRI: Validation and Application in Alzheimer's disease", Elsevier, NeuroImage, Vol. 13, pp. 847-855.

[4] Woo Suk Tae, Sam Soo Kim, Kang Uk Lee, Eui-Cheol Nam and Keun Woo Kim, (2008), "Validation of hippocampal volumes measured using a manual method and two automated methods (FreeSurfer and IBASPM) in chronic major depressive disorder",Neuroradiology Vol.50,pp. 569-581.

[5] A. Ghanei, H. Soltanian-Zadeh, and J. Windham, (1996), A deformable model for hippocampus segmentation: improvements and extension to 3D. In IEEE Nuclear Science Symposium, Vol.3, pp. 1797-1801.

[6] Alexander Hammers,Rolf Heckemann,Matthias J. Koepp,John S. Duncan,Jo V. Hajnal,Daniel Rueckert,Paul Aljabar, (2007), "Automatic detection and quantification of hippocampal atrophy on MRI in temporal lobe epilepsy: A proof-of-principle study", Elsevier, NeuroImage, Vol 36, pp. 38-47.

[7] Bruce Fischl, David H. Salat, Evelina Busa, Marilyn Albert, Megan Dieterich, Christian Haselgrove, Andre van der Kouwe, Ron Killiany, David Kennedy, Shuna Klaveness, Albert Montillo, Nikos Makris, Bruce Rosen,and Anders M. Dale1, (2002), "Whole Brain Segmentation: Automated Labeling of Neuroanatomical Structures in the Human Brain”, Neuron, Vol.33(1): 341-355

[8] Carmichael O.T., Aizenstein H.A., Davis S.W., Becker J.T., Thompson P.M., Meltzer C.C., \& Liu Y.,(2005), "Atlas-based hippocampus segmentation in Alzheimer's disease and mild cognitive impairment" Neuroimage Vol.27 (4), 979-990.

[9] Barnes J., Foster J., Boyes R.G., Pepple T., Moore E.K., Schott, J.M., et al.,(2008), "A comparison of methods for the automated calculation of volumes and atrophy rates in the hippocampus". NeuroImage Vol.40, 1655-1671.

[10] Powell S., Magnotta V.A., Johnson H., Jammalamadaka V.K., Pierson R., Andreasen N.C., (2008), "Registration and machine learning-based automated segmentation of subcortical and cerebellar brain structures". NeuroImage Vol.39, 238-247.

[11] McDonald C.R., Hagler D.J., Ahmadi M.E., Tecoma E., Iragui V., Dale A.M., Halgren E., (2008), "Subcortical and cerebellar atrophy in mesial temporal lobe epilepsy revealed by automatic segmentation". Epilepsy Res. Vol.79, 130-138.

[12] A Computational Approach to Edge Detection by Canny, J., IEEE Trans. Pattern Analysis and Machine Intelligence, 8:679-714, 1986

[13] John Ashburner and Karl J. Friston (June 2000). "Voxel-Based $\quad$ MorphometryTheMethods". NeuroImage 11 (6): $805-$ 821. doi:10.1006/nimg.2000.0582. PMID 10860804. 